\title{
Desarrollo y evaluación de la competencia trabajo en equipo en el grado en Gestión Turística (Primera Fase) $)^{*}$
}

\author{
Elena del Val Noguera ${ }^{1}$, Alberto Palomares Chust $^{1}$, Juan Miguel \\ Alberola Oltra ${ }^{1}$, María Dolores Teruel Serrano ${ }^{1}$, María Manuela \\ Fernández Méndez ${ }^{1}$, Maryland Morant González ${ }^{1}$ y Vicente Benlloch \\ Aparisi $^{1}$
}

${ }^{1}$ Universitat Politècnica de València

\begin{abstract}
The main purpose of this project is to design, evaluate and implement a methodology that allows us to properly develop the competence of Teamwork in the Degree of Tourism Management (GGT) taught at the Gandia Campus of the Universitat Politècnica de València (UPV). In the preliminary phase of the project, which is presented in this paper, we describe the initial experiences developed during 2013-2014 by some teachers who teach in the GGT. In this project, we have collaborated with researchers from the GTI-IA ${ }^{1}$ group of the UPV that have developed a tool for automatic group formation based on the Belbin's Roles Taxonomy. The methodology that we are developing takes as starting point data from the Belbin profiles of students, obtained through surveys conducted by students at the end of a given task in a particular subject. This information is used to establish new work teams to address a new task in the same or in other subjects. The methodology is evaluated through surveys in which students express their satisfaction with respect to the performance of the team after every task. The initial results show that the line we are following seems to be suitable for the development of the teamwork competence. We are currently working on more advanced stages within the framework of a project of the UPV PIME, in order to improve and complete the implementation of this methodology.
\end{abstract}

\footnotetext{
* Proyecto financiado por la Universitat Politècnica de València

${ }^{1}$ http://gti-ia.upv.es/
} 
Desarrollo y evaluación de la competencia trabajo en equipo en el grado en Gestión Turística (Primera Fase)

Keywords: Competence evaluation, Teamwork, Artificial Intelligence, Belbin's Roles, Meaningful Learning.

\begin{abstract}
Resumen
La finalidad principal de este proyecto es diseñar, evaluar e implementar una metodología que nos permita desarrollar adecuadamente la competencia de Trabajo en Equipo en el Grado de Gestión Turística (GGT) que se imparte en la Escuela Politécnica Superior de Gandía (EPSG) de la Universitat Politècnica de València (UPV). En la fase preliminar del proyecto, que es la que presentamos en este artículo, describimos las experiencias iniciales desarrolladas durante el curso 2013-2014 por algunos profesores que impartimos clases en el GGT. Para realizar este trabajo hemos colaborado con un grupo de investigadores del Grupo de Tecnologías Informáticas e Inteligencia Artificial (GTI-IA) de la UPV que han desarrollado unas herramientas para la formación automática de equipos de trabajo óptimos basándose en la Taxonomía de Roles"de Belbin (TRB). La metodología que estamos desarrollando parte de los datos de los perfiles de Belbin de los alumnos, obtenidos mediante encuestas realizadas por los alumnos al finalizar un determinado trabajo en equipo en una determinada asignatura, y utilizamos estas herramientas para establecer nuevos equipos de trabajo para abordar nuevos trabajos en equipo en la misma o en otras asignaturas. La metodología se evalúa mediante encuestas en las que los alumnos expresan su grado de satisfacción con el funcionamiento de los equipos al finalizar cada trabajo. Los resultados iniciales muestran que la línea de trabajo que estamos siguiendo parece bastante adecuada para conseguir los fines previstos al final del proyecto, y actualmente estamos trabajando en fases más avanzadas del proyecto, en el marco de un proyecto PIME de la $U P V$, con el fin de mejorar y terminar de implementar esta metodogías.
\end{abstract}

Keywords: Evaluación Competencias, Trabajo en Equipo, Inteligencia Artificial, Roles de Belbin, Aprendizaje Significativo.

\title{
1 Introducción
}

El Espacio Europeo de Educación Superior (EEES) promueve el uso de aprendizaje basado en entornos colaborativos que fomenten la interacción y participación de distintos alumnos dentro de un equipo de trabajo. En este sentido, es comúnmente aceptado que el aprendizaje colaborativo representa un tema de enorme interés para la comunidad educativa, generando una gran cantidad de estudios e investigaciones. Además, este interés se ha incrementado en los últimos años debido al auge del uso de las tecnologías de la información y comunicación (TICs) como herramientas para apoyar y mejorar la eficacia de este tipo de aprendizaje (Onrubia, Colomina y Engel 2008).

En la literatura podemos encontrar diferentes trabajos que experimentan en las aulas las ventajas del trabajo en equipo (Sáiz y Gómez 2007; Cruz y col. 2007). Un aspecto 
importante en el trabajo en equipo es que los equipos estén formados por estudiantes heterogéneos, con diferentes posibilidades sociales y de aprendizaje que garanticen una riqueza de distintos puntos de vista que propicie la discusión y negociación. Sin embargo, no han aparecido muchos trabajos que se centren en el proceso de formación de equipos, y sobretodo, de equipos heterogéneos.

Christodoulopoulos y Papanikolaou 2007 se presenta una herramienta de formación de equipos heterogéneos y homogéneos basándose en diferentes criterios. La herramienta también permite al profesor modificar los equipos y a los alumnos negociar las agrupaciones. Sin embargo, esta negociación consiste en una interacción directa con el profesor. Graf y Bekele 2006 presentan otra propuesta para formar equipos heterogéneos basándose en los rasgos de los estudiantes. El mecanismo que utilizan trata de maximizar la diversidad del equipo manteniendo un nivel similar de heterogeneidad en todos los equipos. Wang, Lin y Sun 2007 agrupan también estudiantes heterogéneos según su forma de pensar. Al final del proceso, los alumnos evalúan sus equipos mediante cuestionarios. Yannibelli y Amandi 2012 también hacen una propuesta de balancear los distintos tipos de estudiantes que hay en cada equipo.

Aunque estos trabajos tratan el problema de la formación de equipos, utilizan distintas asunciones que pueden ser demasiado restrictivas en contextos educativos reales. En primer lugar, las anteriores propuestas requieren de cierta información previa de los estudiantes, como sus habilidades, atributos que caracterizan a cada uno o tipos diferentes de estudiantes. Además, estos trabajos no utilizan ninguna realimentación de los estudiantes para mejorar la formación de equipos en posteriores iteraciones o proyectos.

El objetivo de nuestro trabajo es por lo tanto, abordar las limitaciones de las aproximaciones actuales. Nuestra propuesta es iterativa, de modo que la formación de los equipos se mejora según se ejecuten más iteraciones, mediante la realimentación obtenida después de cada interacción o proyecto por parte de los alumnos. Además, nuestra propuesta considera la opinión que tienen todos los miembros del equipo sobre un miembro particular, de modo que se emite una opinión consensuada que es más objetiva y realista que la visión personal. Finalmente, la única información previa que se requiere es los distintos tipos en los que se puede clasificar a cada estudiante, pero no cuál es tu tipo concreto ni cuáles son sus habilidades, su forma de trabajar o su forma de actuar.

\section{Objetivos}

El problema que nos planteamos es la formación de equipos de alumnos óptimos para desarrollar y evaluar la competencia trabajo en equipo. En general este problema es bastante complejo de resolver por la dificultad para encontrar los grupos de alumnos adecuados que generen una correcta dinámica de trabajo en los diferentes equipos. Para intentar resolver este problema estamos analizado y evaluando una metodología de formación de grupos basada en la Teoría de Roles de Belbin (TBR). Belbin 2010 hace un profundo estudio sobre la influencia de los roles o tipos de comportamiento de 


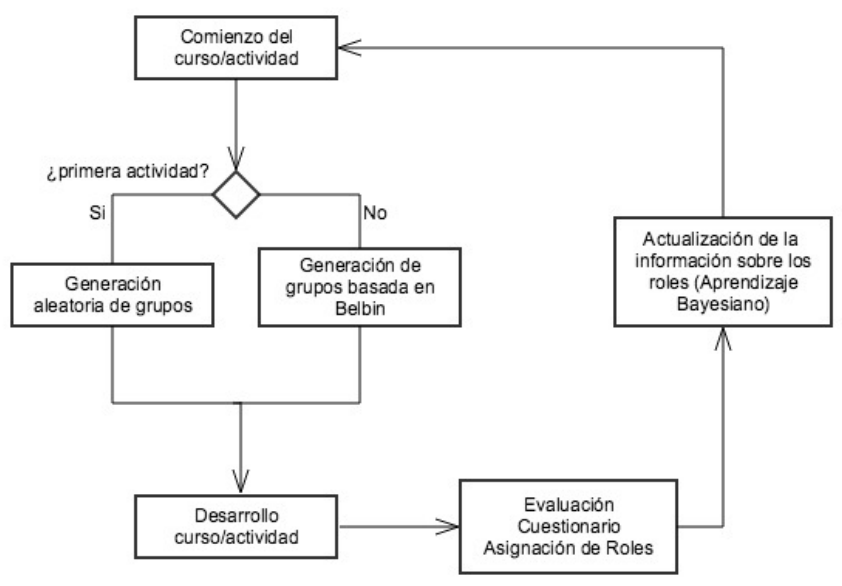

Fig. 1: Etapas del proceso de generación automática de grupos.

las personas que forman un equipo de trabajo y destaca la importancia de la composición de un equipo de trabajo para que el resultado sea satisfactorio. Belbin propone 9 patrones útiles (roles) en un equipo de trabajo. Entre las conclusiones más destacadas de su trabajo podemos remarcar que los equipos con roles poco heterogéneos tienden a producir resultados insatisfactorios, mientras que los equipos de trabajo más eficaces suelen tener una distribución heterogénea de estos perfiles. El objetivo específico de este artículo es presentar la metodología que estamos desarrollando para trabajar la competencia del trabajo en equipo y presentar los primeros resultados obtenidos durante el curso 2013-2014.

\section{Desarrollo de la Innovación}

El principal problema del docente es cómo crear equipos cuando el número de alumnos en el aula es elevado y no se tiene información sobre los perfiles de los alumnos. Durante el curso 2013-2014 hemos empezado a implementar algunas iniciativas para diseñar y evaluar una metodología que nos permita desarrollar el trabajo en equipo de los alumnos del GGT. Los investigadores del GTI-IA han desarrollado una herramienta, basada en técnicas de inteligencia artificial como el aprendizaje automático y la formación de coaliciones, que permite generar grupos de alumnos óptimos, desde el punto de vista de la heterogeneidad de los perfiles de Belbin de los alumnos en los equipos. 
La metodología comienza con una primera fase donde se se generan grupos aleatorios de alumnos para realizar un primer trabajo en grupo (TG) (ver Figura 1). Al finalizar este primer trabajo, hay una segunda fase donde se realiza un proceso de recogida de información sobre el funcionamiento del grupo en general y de cada componente del grupo. Las cuestiones planteadas para la recogida de información se pueden ver en el Anexo A.

Además, en cada grupo, cada miembro del grupo asigna el rol Belbin que considere más adecuado a cada uno de sus compañeros. Los alumnos tienen disponibles las descripciones de los roles de Belbin. Los perfiles/roles propuestos por Belbin en un equipo de trabajo son los siguientes:

- Generador: Solucionan problemas mediante pensamiento creativo y enfoques poco ortodoxos.

- Investigador de recursos: Comunicativos, extrovertidos e investigadores.

- Coordinador: Pensamiento maduro, seguro y generalmente ayudan a promover objetivos y toman parte en el proceso de toma de decisiones.

- Moldeador: Trabajan bien con presión y tienen la energía para superar obstáculos.

- Monitor: Tienden a evaluar todas las opciones con precisión.

- Corporativo: Tienden a ser cooperativos y diplomáticos dentro de un equipo.

- Implementador: Transforman ideas en acciones. Generalmente son disciplinados, eficientes y conservadores.

- Finalizador: Constantemente están buscando errores y descuidos.

- Especialista: Proporcionan habilidades específicas y conocimiento técnico.

En la siguiente etapa, se vuelven a generar los grupos de trabajo basándose en la información recogida en el TG anterior y teniendo en cuenta la estimación de roles de Belbin que se han asignado a cada uno de los alumnos. Para que un equipo sea eficaz, es importante que haya una distribución heterogénea de ellos. Nosotros proponemos el uso de una herramienta que de manera automática a partir de la información sobre los perfiles de Belbin de los alumnos es capaz de generar grupos de trabajo heterogéneos (Alberola y col. 2013). Esta herramienta se utiliza en la generación de los grupos en las sucesivas iteraciones (trabajos en grupo). Una de las ventajas de usar esta herramienta es que los alumnos no son los responsables de formar los equipos de trabajo con lo que se evita que los componentes de los grupos se repitan reiterada y sucesivamente en diferentes asignaturas y cursos. El problema de estos equipos habituales es que no trabajan muchas de las competencias requeridas para realizar trabajos en equipo del máximo nivel competencial y no se ajustan a la forma de trabajo en un contexto laboral. 


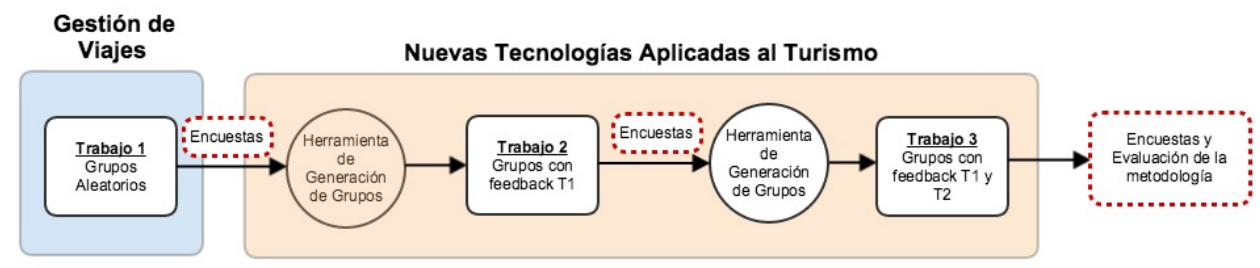

Fig. 2: Diagrama del proceso de aplicación de la generación automática de grupos en el Grado de Gestión Turística.

\subsection{Aplicación en el grado de turismo}

La metodología de generación de grupos ha sido aplicada en el grado de Gestión Turística de la Universitat Politècnica de València. Concretamente, se ha utilizado en las asignaturas Gestión de Viajes (GV) y Nuevas Tecnologías Aplicadas al Turismo (NTAT). Inicialmente hemos generado grupos aleatorios de alumnos para realizar un primer trabajo en grupo en la asignatura GV (ver Figura 2). Al finalizar este primer trabajo los alumnos han realizado las encuestas de caracterización de los perfiles de sus compañeros del trabajo en grupo. Los resultados obtenidos sirvieron para realimentar a la herramienta de generación automática de grupos que basándose en esa información generará los nuevos grupos. Estos nuevos grupos realizaron un segundo trabajo en la asignatura NTAT del segundo cuatrimestre. Después de este trabajo en grupo, los alumnos volvieron a realizar las encuestas de evaluación de perfiles de sus compañeros. Con los resultados obtenidos, la herramienta volvió a generar nuevos grupos de alumnos para realizar un tercer trabajo en grupo en la asignatura NTAT. Además de evaluar a sus compañeros de equipo, al finalizar los trabajos en grupo, los alumnos evaluaron la metodología.

\section{Resultados}

Para evaluar la experiencia utilizando la metodología de generación de grupos basada en los roles de Belbin, se realizaron una serie de preguntas después de cada uno de los trabajos en grupo y una vez acaba la experiencia. En esta sección mostramos algunos de los resultados extraídos una vez procesdas las encuestas (ver gráficas 37). En general, en los resultados se pueden apreciar notables diferencias entre la primera iteración y las otras dos. Podemos estimar que los grupos generados por la herramienta basada en roles de belbin ha mejorado la satisfacción de los alumnos. En las siguientes secciones vemos con más detalle los resultados relacionados con la satisfacción del trabajo en grupo, el éxito en los grupos y la metodología. 


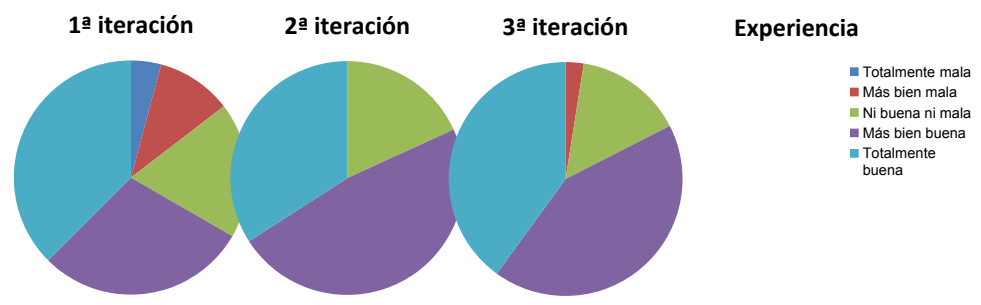

Fig. 3: Evaluación de la experiencia.

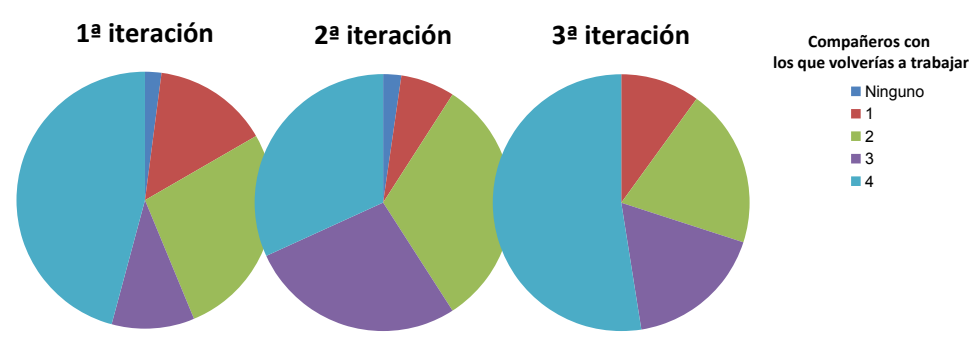

Fig. 4: Compañeros con los que repetirías en el equipo.

\subsection{Grupos}

Uno de los puntos que queríamos evaluar era cómo se ha sentido el grupo con los resultados proporcionados por la metodología propuesta. Para ello tuvimos en cuenta la satisfacción con la experiencia y la satisfacción con los compañeros de los grupos.

Una de las preguntas que se realizó en las encuestas fue sobre la satisfacción con el trabajo realizado en el grupo (ver Figura 3). Si analizamos los resultados positivos (azul y morado), podemos ver que la satisfacción ha ido en aumento, vemos que se ha pasado de una satisfacción que no llega al $70 \%$ a una satisfacción que supera el $80 \%$ en la última iteración.

Conforme se realizan más iteraciones y existe más información sobre los roles de los alumnos, la herramienta proporciona grupos que teóricamente deberían de funcionar mejor. Esto se ve reflejado en el número de compañeros con los que volverían a trabajar. En la primera iteración un $57 \%$ de los alumnos querrían volver a trabajar con todos o casi todos de sus compañeros. Esta cantidad va aumentado en las iteraciones hasta la tercera iteración donde se alcanza un $70 \%$. 


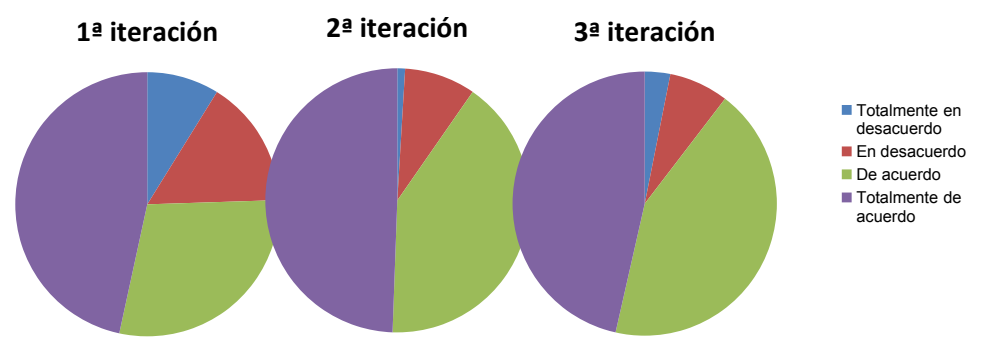

Fig. 5: Aspectos Positivos.

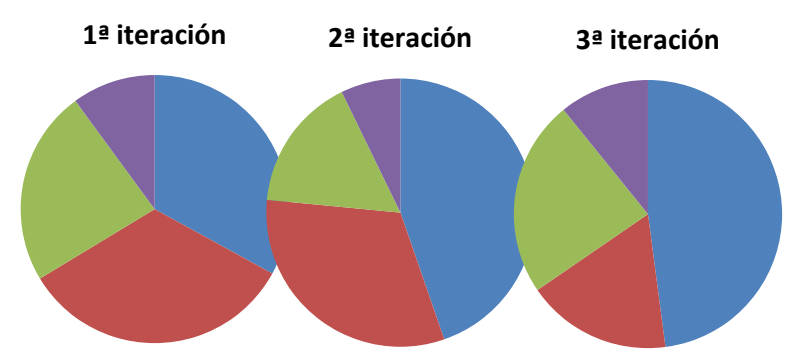

Fig. 6: Aspectos negativos.

\section{2 Éxito}

Otro aspecto que queríamos valorar era el éxito de los grupos. Para ello, asociamos una serie de factores que podrían considerarse como características de un trabajo positivo en el grupo (si hubo buena organización, buena comunicación, respeto de los miembros, si los miembros han hecho su parte del trabajo, si se han escuchado las opiniones de todos...). Las respuestas asociadas a los aspectos positivos de los grupos se muestran en la Figura 5. En las gráficas podemos ver que el funcionamiento de los grupos ha ido a mejor, pasando de un $75 \%$ de alumnos que consideran que su grupo ha funcionado bien hasta un $90 \%$ en las otras dos iteraciones posteriores.

También hemos querido analizar los aspectos que caracterizan negativamente el funcionamiento del grupo (si ha habido miembros cerrados de mente, si ha sido difícil llegar a conclusiones, si ha habido poca paciencia, si alguien no se ha implicado...). En este caso, la mejor iteración fue la segunda, con un $76 \%$ de alumnos que no consideran aspectos negativos frente a un 60-65\% de la primera y la tercera iteración. Sin embargo, si nos fijamos sólo en el azul (totalmente en desacuerdo), tenemos un $48 \%$ de alumnos que han marcado aspectos negativos como "totalmente en desacuerdo". 


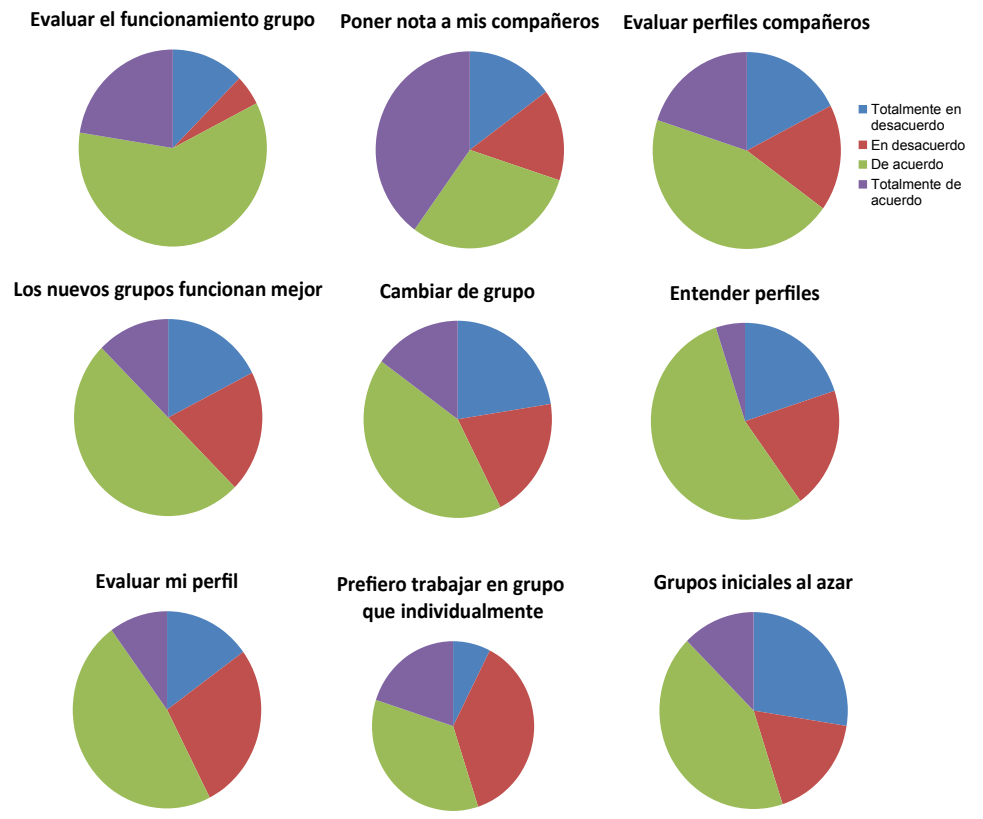

Fig. 7: Evaluación sobre un conjunto de items.

\subsection{Metodología}

Un conjunto de los resultados tenían como objetivo mostrar cómo han visto la experiencia de trabajar con la metodología propuesta. Aspectos como tener la posibilidad de evaluar el funcionamiento del grupo así como poner nota a sus compañeros y evaluar los perfiles de sus compañeros han sido valorados positivamente. Algo que no les ha gustado, por ejemplo, ha sido que los grupos iniciales fuesen al azar.

Otra de las preguntas de la encuesta fue la preferencia a trabajar individualmente o en grupo. Un $45 \%$ de los alumnos afirmó que preferían trabajar individualmente y no en grupo. Esto es un problema, puesto que lo que van a encontrarse en la realidad es el trabajo en grupo, y habría que fomentar esta idea en las distintas asignaturas del grado.

En la encuesta, a la pregunta qué factores consideran importantes a la hora de trabajar en un grupo, la mayoría del alumnado contestó que el factor clave es que haya una buena coordinación y que haya una buena distribución de tareas. Factores que consideran menos importantes son que haya un buen líder o que tengan las mismas aspiraciones de nota.

También se les preguntó por los conflictos idiomáticos así como aspectos a mejorar y aspectos buenos de sus grupos. Simplemente remarcar un aspecto que nos llamó la atención y es que los alumnos suelen percibir que todos los miembros han trabajado por igual o que ellos han trabajado más que el resto, pero casi nadie percibe que haya 
trabajado menos que el resto. Esto es muy curioso porque de 150 respuestas, sólo 2 han dicho que reconocen que son los que menos o de los que menos han trabajado de su equipo.

\section{Discusion y Conclusiones}

Durante la aplicación de la metodología de generación de grupos basado en Belbin se dectaron una serie de problemas que esperamos ir corrigiendo en las próximas fases del PIME. Entre los problemas detectados encontramos los siguientes:

- En algunos grupos la mayoría de sus miembros son estudiantes muy motivados y en otros la mayoría son estudiantes más desinteresados en un mismo grupo. La herramienta tiene en cuenta los roles de los alumnos pero no su nivel de trabajo y esfuerzo personal. En futuras fases, nos planteamos añadir criterios objetivos a la hora de generar los grupos. Esto nos permitirá poder equilibrar el número de estudiantes teniendo en cuenta su rendimiento.

- Varios extranjeros en un mismo grupo. En el caso del grado de Turismo hay muchos estudiantes extranjeros y con el objetivo de fomentar su integración la herramienta debería considerar restricciones que impidan que haya grupos donde la mayoría de los estudiantes son extranjeros.

- En algunas situaciones han aparecido varios líderes en un mismo grupo. Esto ha generando conflictos en la toma de decisiones.

- Otro de los problemas que les han surgido a los alumnos es la dificultad para quedar fuera de las horas de clase. A la hora de formar los grupos, se tendría que tener en cuenta la compatibilidad de horarios para facilitar las reuniones fuera de las horas de clase.

- Existe una preocupación por los alumnos con mayor rendimiento por el desinterés o incumplimiento de sus tareas de otros compañeros.

En base a estos problemas detectados, planteamos un conjunto de propuestas de mejora:

- Introducir restricciones en la herramienta de generación de grupos para intentar que:

- el nivel de los alumnos sea más heterogéneo. Esto se podría hacer en base a las notas obtenidas en la/s asignatura/s;

- no coincida más de un extranjero por grupo;

- los miembros de un mismo grupo compartan el mismo grupo de prácticas. 
- Sesión pedagógica con los alumnos antes de empezar los trabajos en grupo para explicar: (i) el proyecto que se va a desarrollar en las asignaturas; (ii) la importancia del trabajar en equipo; y (iii) la importancia de la heterogeneidad de los equipos. Para esta propuesta se plantea invitar a un directivo de empresa con experiencia en gestión de equipos para motivar a los alumnos.

En base a la experiencia que hemos tenido utilizando la metodología propuesta también nos planteamos otras mejoras:

- Trasladar el feedback sobre los resultados y los roles (análisis individual y sobre cómo es percibido cada alumno por sus compañeros) a los alumnos tras cada iteración (trabajo en grupo).

- Estudiar modelos basados en personalidad como otro criterio para formar los grupos en lugar de utilizar Belbin.

- En el caso de seguir utilizando Belbin como criterio de formación de grupos, revisaremos el número de roles para adaptarlo al contexto del aula. También tendremos en cuenta la posibilidad de que una persona pueda jugar más de un rol.

- Ampliar el proyecto al segundo curso en la asignatura Recursos territoriales y en el tercer curso a Gestión de Viajes, Nuevas tecnologías y OGEII.

Los resultados preliminares obtenidos durante el curso 2013-2014 muestran que el grado de satisfacción de los alumnos con sus equipos de trabajo tiende, en promedio a mejorar en los sucesivos trabajos en grupo. Consideramos que este resultado es importante ya que nos está indicando que la metodología que estamos desarrollando parece que es adecuada para desarrollar la competencia trabajo en equipo de una manera más satisfactoria. También tenemos que destacar que muchos alumnos no entienden que los equipos de trabajo se formen de esta manera. En general es más difícil trabajar con compañeros no habituales y lógicamente los alumnos preferirían formar ellos los equipos para así poder trabajar con sus compañeros habituales. Por lo tanto es necesario explicarles mejor que el propósito de estas metodologías es que desarrollen competencias adicionales para poder realizar los trabajos en grupo de máximo nivel. 


\section{Agradecimientos}

Este trabajo ha sido subvencionado por el Proyecto de Innovación y Mejora Educativa PIME B-08/14 titulado: Desarrollo y evaluación de la competencia de trabajo en equipo en el grado en Gestión Turística.

\section{A Anexo: Encuestas}

- Valoración del grupo:

- ¿Cómo evaluarías la contribución de tu compañero al equipo?

- El/Ella es una persona trabajadora

- El/Ella ha trabajado más que otros miembros del equipo

- El/Ella ha trabajado como el resto de miembros del equipo

- El/Ella ha trabajado menos que el resto de miembros del equipo

- El/Ella es el miembro del equipo que menos a trabajado

- ¿Qué puntuación asignarías a tu compañero de equipo (en el rango del 1 al 10)?

- ¿Cómo calificas tu experiencia dentro de este grupo de trabajo?

- ¿Con cuántos miembros de tu grupo de trabajo te gustaría volver a trabajar?

- Valora las siguientes afirmaciones según en grado en que estés de acuerdo con ellas (Totalmente en desacuerdo, En desacuerdo, De acuerdo, Totalmente de acuerdo)

- Han habido normas y tareas claras para todos

- Cada miembro ha hecho su parte del trabajo

- Las actitudes de los miembros han sido buenas

- Se han cumplido los plazos que hemos ido estableciendo para la actividad

- El trabajo realizado es satisfactorio para el grupo

- Los miembros han aceptado las críticas de forma positiva

- Se han escuchado las opiniones de todos

- Algunos miembros son cerrados de mente ante las sugerencias de otros

- Los miembros han tratado a los demás con respeto

- Ha habido una buena coordinación

- Ha sido dificil llegar a decisiones dentro del grupo

- En las discusiones del grupo, con facilidad nos hemos desviado del tema principal 
- Me he sentido a gusto trabajando en este equipo

- Ha existido un clima de cooperación

- Me he sentido que he aportado cosas a este equipo

- Considero que ha faltado organización dentro del grupo

- Los miembros de mi grupo han tenido poca paciencia

- He tenido la sensación de no saber hacia dónde iba el trabajo

- Apreciación general:

- En comparación con los otros compañeros de tu equipo, ¿cómo crees que has trabajado tú?

- Soy el que más o de los que más han trabajado

- Algunos han trabajado más que yo

- Hemos trabajado todos por igual

- Algunos han trabajado menos que yo

- Soy el que menos o de los que menos han trabajado

- ¿Cuál ha sido la lengua utilizada en el grupo?

- Cita brevemente aspectos mejorables de tu equipo (organización, reparto de trabajo, comunicación, etc.)

- Cita brevemente aspectos positivos de tu equipo (organización, reparto de trabajo, comunicación, etc.)

- El idioma utilizado ha supuesto una barrera para el trabajo en grupo

- Valoración del método:

- ¿Qué es lo que más te ha gustado/más valoras del método que hemos seguido? (Totalmente en desacuerdo, En desacuerdo, De acuerdo, Totalmente de acuerdo)

- Seleccionar Aleatoriamente los Grupos la primera vez

- Entender los Perfiles de Belbin

- Evaluar mi propio Perfil de Belbin (encuesta inicial)

- Evaluar los Perfiles de Belbin de mis Compañeros de Grupo (encuesta final)

- Evaluar la Calidad del Trabajo de mis Compañeros (Poner Nota)

- Evaluar el funcionamiento general del Grupo

- Cambiar varias veces los Grupos durante el Curso

- Los nuevos grupos funcionan mejor

- Sobre el trabajo en grupo: Prefiero trabajar en grupo que individualmente 
- Consideras que para trabajar en grupo es necesario reunirse o trabajar en red

- Qué consideras más importante para el trabajo en grupo:

- Un buen ambiente

- Una buena distribución del trabajo

- Un buen líder

- Una buena comunicación

- Que todos tengamos las mismas aspiraciones de nota

\section{Referencias bibliográficas}

Alberola, J. M. y col. (2013). "Simulating a collective intelligence approach to student team formation". En: International Conference on Hybrid Artificial Intelligence Systems. Vol. 8073, págs. 161-170.

Belbin, Raymond M (2010). Team roles at work. Routledge.

Christodoulopoulos, Christos E. y Kyparisia A. Papanikolaou (2007). "A Group Formation Tool in an E-Learning Context". En: 2012 IEEE 24th International Conference on Tools with Artificial Intelligence 2, págs. 117-123. ISSN: 1082-3409.

Cruz, A y col. (2007). "Hacia la convergencia europea: relato de una experiencia de innovación docente en la UEM". En: Revista Iberoamericana de Educación 43.1, pág. 6.

Graf, Sabine y Rahel Bekele (2006). "R.: Forming Heterogeneous Groups for Intelligent Collaborative Learning Systems with Ant Colony Optimization". En: Berlin / Heidelberg. Springer, págs. 217-226.

Onrubia, Javier, Rosa Colomina y Anna Engel (2008). "Los entornos virtuales de aprendizaje basados en el trabajo en grupo y el aprendizaje colaborativo". En: Coll, C. E Monereo, C.(Coords.). Psicología de la Educación Virtual, págs. 233-252.

Sáiz, María Soledad Ibarra y Gregorio Rodríguez Gómez (2007). "El trabajo colaborativo en las aulas universitarias: reflexiones desde la autoevaluación". En: Revista de Educación 344, págs. 229-230.

Wang, Dai-Yi, Sunny S.J. Lin y Chuen-Tsai Sun (2007). "DIANA: A computersupported heterogeneous grouping system for teachers to conduct successful small learning groups". En: Computers in Human Behavior 23.4, págs. 1997-2010. ISSN: 0747-5632. 
A. Palomares, M.D. Teruel, M.M Fernández, J.M Alberola, E. del Val, M. Morant, V. Benlloch

Yannibelli, Virginia y AnalÃa Amandi (2012). "A deterministic crowding evolutionary algorithm to form learning teams in a collaborative learning context". En: Expert Systems with Applications 39.10, págs. 8584-8592. ISSN: 0957-4174. 